
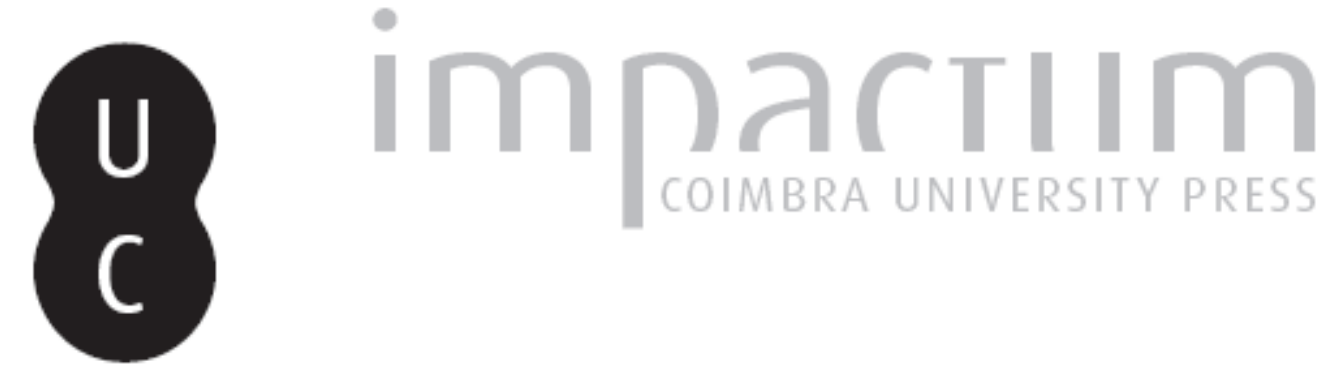

\title{
Análise de tendência da precipitação anual e mensal no período 1900-2000, em Portugal Continental
}

\author{
Autor(es): $\quad$ Lima, M. Isabel P. de; Marques, Ana C.; Lima, João L. M. P. de \\ Publicado por: Associação Portuguesa de Riscos, Prevenção e Segurança \\ URL \\ persistente: \\ URI:http://hdl.handle.net/10316.2/39088 \\ DOI: \\ DOI:http://dx.doi.org/10.14195/1647-7723_12_2 \\ Accessed : $\quad$ 26-Apr-2023 05:41:58
}

A navegação consulta e descarregamento dos títulos inseridos nas Bibliotecas Digitais UC Digitalis, UC Pombalina e UC Impactum, pressupõem a aceitação plena e sem reservas dos Termos e Condições de Uso destas Bibliotecas Digitais, disponíveis em https://digitalis.uc.pt/pt-pt/termos.

Conforme exposto nos referidos Termos e Condições de Uso, o descarregamento de títulos de acesso restrito requer uma licença válida de autorização devendo o utilizador aceder ao(s) documento(s) a partir de um endereço de IP da instituição detentora da supramencionada licença.

Ao utilizador é apenas permitido o descarregamento para uso pessoal, pelo que o emprego do(s) título(s) descarregado(s) para outro fim, designadamente comercial, carece de autorização do respetivo autor ou editor da obra.

Na medida em que todas as obras da UC Digitalis se encontram protegidas pelo Código do Direito de Autor e Direitos Conexos e demais legislação aplicável, toda a cópia, parcial ou total, deste documento, nos casos em que é legalmente admitida, deverá conter ou fazer-se acompanhar por este aviso.

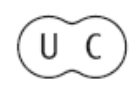




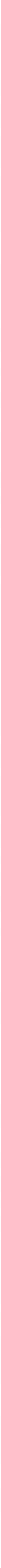




\title{
Análise de tendência da precipitação anual e mensal no período 1900-2000, em Portugal Continental
}

\author{
M. Isabel P. de Lima ${ }^{1.2}$ \\ Ana C. Marques ${ }^{1,4}$ \\ João L. M. P. de Lima ${ }^{1,3}$
}

\begin{abstract}
Resumo:
Este trabalho debruça-se sobre o estudo da precipitação em Portugal Continental, com base em nove séries temporais de observações udométricas, abrangendo todo o século XX. Os dados analisados têm uma distribuição geográfica que cobre várias regiões do País. As séries são analisadas por métodos estatísticos com o objectivo de testar se existe uma alteração, ao longo do tempo, da grandeza climática em estudo. É feita a análise de tendência da precipitação anual e da precipitação mensal, o que permite caracterizar, neste último caso, as alterações da distribuição da precipitação durante 0 ano, no território nacional.

Palavras chave:

Precipitação, tendência, alterações climáticas, sazonalidade, Portugal Continental.
\end{abstract}

\section{Résumé:}

Dans cet article, on analyse avec des méthodes statistiques les tendances de données pluviométriques annuelles et mensuelles enregistrées à 9 endroits au Portugal continental, dans la période 1900-2000. Cet étude permit de caractériser des changements de la distribution de la précipitation pendant l'année, observée dans le vingtième siècle au Portugal continental.

Mots clés:

Précipitation, tendance, changement climatique, saisonnalité, Portugal continental.

\section{Abstract:}

This work investigates the presence of trends in the temporal structure of precipitation in Mainland Portugal. The study uses annual and monthly precipitation data from 1900 to 2000 , recorded in 9 locations scattered over the territory. The data are analyzed using statistical methods (e.g. Mann-Kendall trend test). In order to take into account seasonality and serial correlation, the different months of the year were analysed separately. The analyses of monthly data lead to a characterization of changes in the distribution of precipitation within the year over Mainland Portugal.

Key words:

Precipitation, trend, climate change, seasonality, Mainland Portugal.

\section{Introdução}

Nas últimas décadas, as variações climáticas têm sido analisadas com especial atenção, interessando os resultados desses estudos a variadíssimas áreas de investigação. São inúmeras as potenciais implicaçõès das variações climáticas no meio ambiente, nos ecossistemas, na gestão dos recursos, nas actividades económicas e, portanto, no nosso dia-a-dia.

O estudo do sistema climático faz muitas vezes uso de modelos complexos que pretendem, por exemplo,

1 IMAR, Instituto do Mar - Centro Interdisciplinar de Coimbra.

2 Departamento Florestal da Escola Superior Agrária do Instituto Politécnico deCoimbra-Bencanta, 3040-316 Coimbra, Portugal. Tel.: +351.239.802.940; Fax: +351.239.802.979; iplima@mail.esac.pt.

3 Departamento de Engenharia Civil da Faculdade de Ciência e Tecnologia da Universidade de Coimbra.

4 Bolseira da Fundação para a Ciência e a Tecnologia descrever e prever cenários de alterações climáticas. A utilização destas ferramentas passa, no entanto, pela análise de séries temporais de dados empíricos relevantes (e.g. quantidade e distribuição da precipitação, temperatura do ar e temperatura da água do mar), que fornece informação essencial para uma melhor compreensão e caracterização dos processos envolvidos. De entre estes dados destaca-se a precipitação e a investigação de tendências neste processo. A presença de tendência em séries de precipitação resulta essencialmente da variabilidade natural presente nos processos hidrológicos ou está directamente relacionada com a existência de alterações climáticas.

Neste contexto, este trabalho debruça-se sobre o estudo da precipitação em Portugal Continental, combase em 9 séries temporais longas de observações udométricas, abrangendo todo o século XX. Os dados 
analisados têm uma distribuição geográfica por várias regiões doPaís. As séries são analisadas por métodos estatísticos com o objectivo de testar, neste período, a presença de tendência da precipitação anual e da precipitação mensal. A análise dos dados mensais permite caracterizar as alterações da distribuição da precipitação durante o ano, no território nacional.

\section{Dados udométricos}

As observações udométricas utilizadas neste estudo estiveram a cargo do Instituto de Meteorologia (IM) e do Instituto da Água (INAG). São usados dados mensais e anuais recolhidos em 9 localizações espacialmente dispersas sobre Portugal Continental (Figura 1), o que torna as séries representativas de diferentes regimes de precipitação que afectam o território. As séries escolhidas abrangem todo o século XX.

O Quadro I apresenta dados sobre a localização dos 9 pontos de medição, período estudado, e correspondente precipitação anual média e coeficiente de variação neste período. A Figura 2 mostra, como exemplo, as séries de precipitação anual, mensal e precipitação média mensal para o Porto, Lisboae Beja. A precipitação média mensal para todas as estações estudadas encontra-se representada na Figura 3.

O Quadro I e as Figuras 2 e 3 evidenciam as diferenças norte/sul e este/oeste observadas no regime de precipitação em Portugal Continental. Este regime é caracterizado por variabilidades temporal e espacial acentuadas (e.g. de LIMA, 1998; de LIMA et al., 2002, MIRANDA et al. 2002, de LIMA et al., 2005), destacando-se também uma grande variabilidade inter-anual e sazonal. Tal como as restantes regiões do Sul da Europa, Portugal tem um clima tipicamente mediterrâneo, com Verões secos e quentes, mais pronunciados no Sul do país. A sazonalidade do regime pluviométrico diz respeito quer à quantidade total de precipitação quer à sua natureza (frontal ou convectiva)e extensão temporal e espacial. A precipi- tação tipo convectiva é frequente durante o Verão, princípio e meio do Outono, predominantemente nas regiões do sul; a precipitação frontal ocorre principalmente no Inverno e afecta principalmente o norte de Portugal Continental.Entreos dados analisados, a estação mais chuvosa, Porto, localiza-se no litoral norte de Portugal Continental, com uma precipitação anual média de $1204 \mathrm{~mm}$; as estações menos chuvosas, Lagos e Moncorvo, localizam-se no Algarve (região sul) e no Nordeste Transmontano, com precipitação anual média de $526 \mathrm{~mm}$ e $564 \mathrm{~mm}$, respectivamente. Os dados que apresentam maior coeficiente de variação são Lagos e São Brás de Alportel, no Algarve (Quadro I). Em média, o mês mais chuvoso foi Dezembro e o mês mais seco foi Julho; o trimestre mais chuvoso foi de Novembro a Janeiro e o mais seco de Junho a Agosto, em que se verificaram, respectivamente, cerca de 41 e $6 \%$ da precipitação anual.

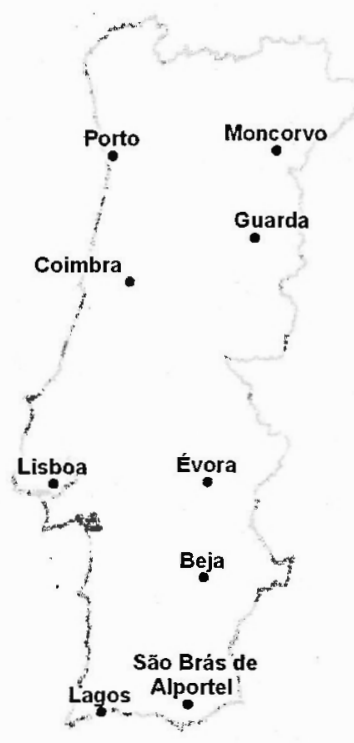

Figura 1 - Localização das estações meteorológicas em Portugal Continental.

\begin{tabular}{|c|c|c|c|c|c|c|}
\hline Estação & $\begin{array}{c}\text { Latitude } \\
(\mathbf{N})\end{array}$ & $\begin{array}{c}\text { Longitude } \\
(\mathbf{W})\end{array}$ & $\begin{array}{c}\text { Altitude } \\
(\mathbf{m})\end{array}$ & $\begin{array}{c}\text { Periodo } \\
\text { estudado }\end{array}$ & $\begin{array}{c}\text { Prec.anual } \\
\text { média (mm) }\end{array}$ & $\begin{array}{c}\text { Coef. } \\
\text { variação }\end{array}$ \\
\hline 546 Porto & $41^{\circ} 08^{\prime}$ & $8^{\circ} 36^{\prime}$ & 93 & $1900-2000$ & 1204.3 & 0.221 \\
\hline $060 / 04$ Moncorvo & $41^{\circ} 10^{\prime}$ & $7^{\circ} 03^{\prime}$ & 385 & $1900-2000$ & 563.5 & 0.252 \\
\hline 082 Guarda & $40032^{\prime}$ & $7016^{\prime}$ & 119 & $1900-2000$ & 1020.1 & 0.282 \\
\hline 549 Coimbra & $40^{\circ} 12^{\prime}$ & $8^{\circ} 25^{\prime}$ & 141 & $1900-1995$ & 979.5 & 0.218 \\
\hline 535 Lisboa & $38^{\circ} 43^{\prime}$ & $9^{\circ} 09^{\prime}$ & 77 & $1900-2000$ & 725.5 & 0.243 \\
\hline 557 Évora & $38^{\circ} 34^{\prime}$ & $7^{\circ} 54^{\prime}$ & 309 & $1900-2000$ & 631.8 & 0.249 \\
\hline 562 Beja & $38^{\circ} 01^{\prime}$ & $7^{\circ} 52^{\prime}$ & 246 & $1900-2000$ & 568.9 & 0.240 \\
\hline 31E/01 Lagos & $37^{\circ} 06^{\prime}$ & $8^{\circ} 40^{\prime}$ & 14 & $1902-2000$ & 525.5 & 0.302 \\
\hline 31J/01 São Brás de Alportel & $37^{\circ} 10^{\prime}$ & $7^{\circ} 45^{\prime}$ & 325 & $1901-2000$ & 832.2 & 0.305 \\
\hline
\end{tabular}

Quadro I - Informação sobre as séries temporais de precipitação de Portugal Continental analisadas: identificação, coordenadas geográficas e altitude das estações; duração das séries; precipitação anual média e coeficiente de variação da precipitação anual. 

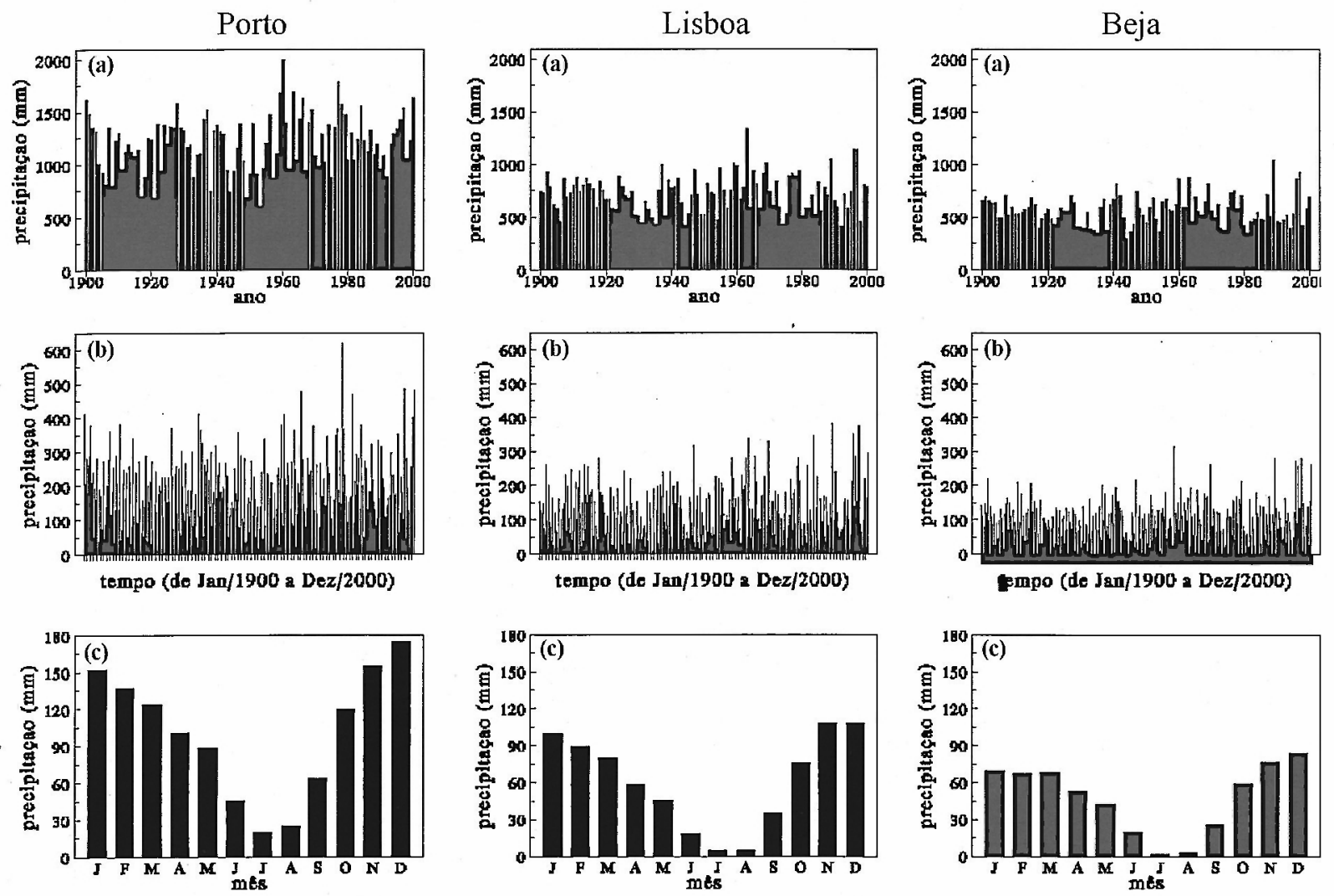

Figura 2 - Séries da precipitação (em mm) no período 1900-2000 no Porto, Lisboa e Beja:

(a) precipitação anual; (b) precipitação mensal; (c) precipitação mensal média.

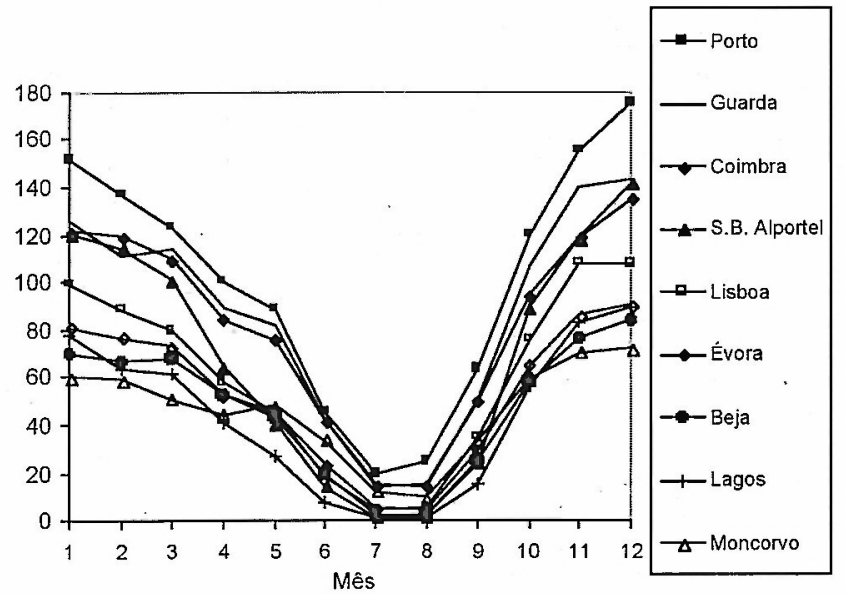

Figura 3 - Precipitação média mensal em vários locais em Portugal Continental (ver Quadro I).

\section{Aspectos metodológicos}

Os testes estatísticos de tendência têm como pressuposto a independência dos dados. Assim, tornase necessário testar a existência de correlação nos dados para assegurar a fiabilidade dos resultados do teste de tendência. Dos testes recomendados pela Organização Meteorológica Mundial (e.g. SNEYERS, 1990), utilizou-se neste trabalho o teste não paramétrico de Wald-Wolfowitz (WALD e WOLFOWITZ, 1943).

Seja $x_{1}, x_{2}, \ldots, x_{n}$ uma amostra aleatória com valor médio nulo. A estatística de teste $U$ é definida por,

$$
U=\frac{1+(n-1)\left[\frac{\sum_{i=1}^{n} x_{i} x_{i+1}}{\sum_{i=1}^{n} x_{i}^{2}}\right]}{\sqrt{n-1}}
$$

em que $x_{\mathrm{i}}$ é a observação de ordem i, $n$ é o tamanho da amostra, e $x_{n+1}=x_{1}$.

Sob a hipótese nula $\mathrm{H}_{0}$ de não existência de correlação, a estatística de teste $U$ segue uma distribuição Normal reduzida (valor médio nuloe variância unitária). Rejeitamos $\mathrm{H}_{0}$ para o nível de confiança, por exemplo, de $95 \%$, se

$$
\mathrm{P}(U<u)=\Phi(u)>0.95
$$

em que u é o valor observado da estatística $U$. 
A existência de correlação introduz erros que conduzem frequentemente à rejeição da hipótese nula, sem que exista evidência para tal, isto é, conduz frequentemente à aceitação de tendência quando esta na realidade não se verifica (SNEYERS, 1990).

Para testar a tendência das séries de precipitação mensal e anual, recorremos ao teste não-paramétrico de Mann-Kendall (e.g. GLLBERT, 1997), Originalmente criado por MANN (1945) e reformulado por KENDALL (1948), este teste tem a vantagem de não assumir nenhuma distribuição subjacente aos dados. Este teste é recomendado pela Organização Meteorológica Mundial (e.g. SNEYERS, 1990) e tem vindo a ser largamente aplicado.

Seja $x_{1}, x_{2}, \ldots, x_{\mathrm{n}}$ uma amostra aleatória. A estatística de teste $R$ é definida por

$$
R=\sum_{i=1}^{n} p_{i}
$$

com $p_{i}$ igual ao número de termos de ordem superior cujo valor excede $x$.

Sob a hipótese nula de não existência de correlação, a estatística $R$ segue uma distribuição Gaussiana (Normal), com média e variância dados, respectivamente, por

$$
\begin{aligned}
& E(R)=\frac{n(n-1)}{4} \\
& \operatorname{Var}(R)=\frac{n(n-1)(2 n+5)}{72}
\end{aligned}
$$

Definindo

$$
Z=\frac{r-E(r)}{\sqrt{\operatorname{Var}(r)}} \cap N(0,1)
$$

em que $r$ é ovalor observado da estatística $\mathrm{R}$, rejeitamos a hipótese nula $\mathrm{H}_{0}$, de não existência de tendência, para um nível de confiança, por exemplo, de $95 \%$, se $\Phi(|z|)>0.95$.

Quandoa estatística de testeé significante, verifica-se uma tendência positiva (aumento) ou negativa (diminuição) da precipitação, em função do sinal do valor observado $z$, da estatística $Z$ (respectivamente, positivo ou negativo).

Para estimar o valor da tendência (por exemplo, em $\mathrm{mm} /$ década) foi utilizado o método não-paramétrico de Sen (SEN, 1968; GILBERT, 1987), onde se assume que a tendência é linear. Isto significa que a série temporal de precipitação, $P(t)$, pode ser descrita por um modelo da forma

$$
P(t)=Q t+B
$$

onde $Q$ é a tendência (declive da recta) e $B$ é uma constante.
Para estimar a tendência $Q$, primeiro calculam-se todos os declives (tendência) entre cada par de variáveis $j$ e $k$.

$$
Q_{i}=\frac{x_{j}-x_{k}}{j-k} \operatorname{com} j>k
$$

Se existirem $n$ valorespara $x_{j}$, temos $N=\frac{n(n-1)}{2}$ estimativas para a tendência $Q_{\dot{\mathrm{H}}}$ O $\mathrm{O}$ estimador de Sen para a tendência é a mediana dos $N$ valores obtidos para $Q_{\mathrm{i}}$. Depois de ordenados os $N$ valores de $Q_{\mathrm{i}}$; por ordem crescente, o estimador de Sen para a tendência é

$$
\left\{\begin{array}{l}
Q=Q_{[(N+1) / 2]}, \text { se } N \text { é impar } \\
Q=\frac{1}{2}\left(Q_{[N / 2]}+Q_{[(N+2) / 2], \text { se } N \text { é } \operatorname{par}(9 \mathrm{~b})}\right.
\end{array}\right.
$$

\section{Resultados e discussão}

Foram testadas a consistência e homogeneidade das séries de precipitação anual, não havendo evidência deste tipo de problemas nos dados. Os ensaios de correlação e tendência foram aplicados aos dados anuais e mensais da precipitação devido à forte sazonalidade presente na precipitação em Portugal Continental. Nos testes de tendência foramconsiderados vários níveis de confiança: 90, 95, 99 e 99.9\%. Para os diferentes tipos de dados determinou-se o estimador de tendência de Sen.

\section{Precipitação anual}

Os resultados do teste de Wald-Wolfowitz (teste de correlação) para as séries anuais indicam existência de correlação em seis das nove estações estudadas (Quadro II). Assim sendo, ao analisarmos os resultados do teste de tendência temos de ter em conta que estes poderão estar enviesados. A existência de correlação conduz frequentemente à existência de tendência sem que de facto exista evidência para tal.

\begin{tabular}{|l|c|c|c|}
\hline \multicolumn{1}{|c|}{ Estação } & $\mathbf{u}$ & $\mathbf{P}(U<\boldsymbol{u})$ & Decisão \\
\hline Porto & 2,23 & 0,9872 & Rejeitar $\mathrm{H}_{\mathbf{0}}$ \\
\hline Moncorvo & 2,56 & 0,9948 & Rejeitar $\mathrm{H}_{0}$ \\
\hline Guarda & 1.74 & 0,9587 & Rejeitar $\mathrm{H}_{\mathbf{0}}$ \\
\hline Coimbra & 0,97 & 0,8238 & Não rejeitar $\mathrm{H}_{0}$ \\
\hline Lisboa & 1,61 & 0,9462 & Não rejeitar $\mathrm{H}_{0}$ \\
\hline Évora & 1,73 & 0,9580 & Rejeitar $\mathbf{H}_{0}$ \\
\hline Beja & 2,19 & 0,9857 & Rejeitar $\mathrm{H}_{0}$ \\
\hline Lagos & 2,47 & 0,9932 & Rejeitar $\mathbf{H}_{0}$ \\
\hline São Brás de Alportel & 1,36 & 0,9131 & Não rejeitar $\mathrm{H}_{0}$ \\
\hline
\end{tabular}

Quadro II - Resultados do teste de Wald-Wolfowitz para as séries de precipitação anual, para um nível de confiança de $95 \%$. 
Para um nível de confiança de $90 \%$, o teste de Mann-Kendall aplicado às séries anuais revelou a não existência de tendência na precipitação anual nas nove estações em estudo, em Portugal Continental (Figura 4). Este comportamento foi também observado por SERRANO et al. (1998) no período 1921-1995, em 4 das 9 estações agora estudadas (Porto, Coimbra, Lisboa e Évora); o estudo incide sobre a existência de tendência da precipitação na Península Ibérica, baseado na análise de 40 séries anuais (4 destas séries são as acima referidas, localizadas em território nacional).

A Figura 5 mostra as séries de precipitação anual, a recta de tendência, e os resíduos (diferenças relativamente à precipitação anual média). $O$ declive da recta de tendência, determinado com o método não-paramétrico de Sen, é apresentado no Quadro III; no entanto, como já foi referido, esta tendência não é estatisticamente significativa. Neste Quadro, valores positivos indicam uma tendência crescente da precipitação e valores negativos indicam uma tendência decrescente da precipitação.
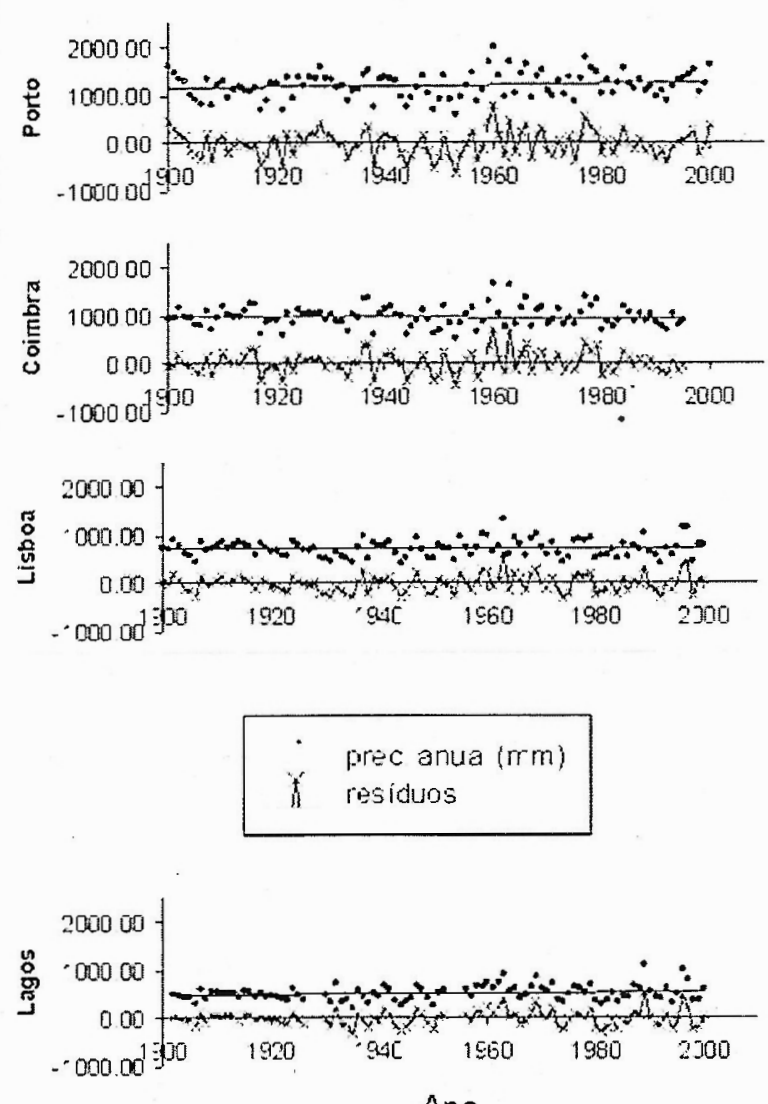

Ano
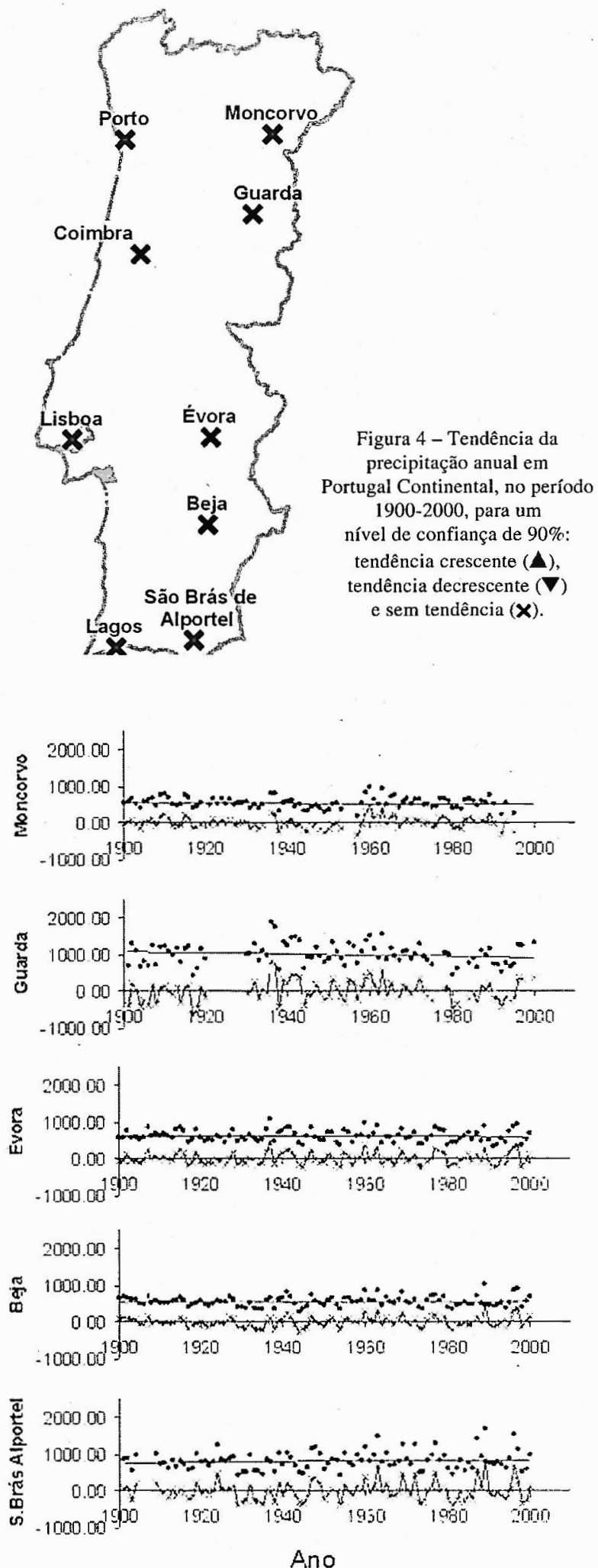

Fig. 5 - Séries da precipitação anual (em mm) no período 1900-2000 e respectiva recta de tendência com declive obtido com o estimador de Sen; e correspondentes resíduos (em $\mathrm{mm}$ ) da precipitação anual relativamente à média. 


\section{Precipitação mensal}

As séries de precipitação mensal não apresentam correlação. A ausência de correlação deve-se ao modo como as séries mensais foram construídas. Uma vez que cada série contém dados correspondentes a um mês do ano, entre dois dados consecutivos existe um intervalo de onze meses que é suficientemente longo para os dados serem independentes.

Utilizando o teste de tendência de Mann-Kendall, para um nível de significância de $5 \%$, verificou-se um decréscimo generalizado no mês de Março, como se pode visualizar na Figura 6 . Esta tendência foi

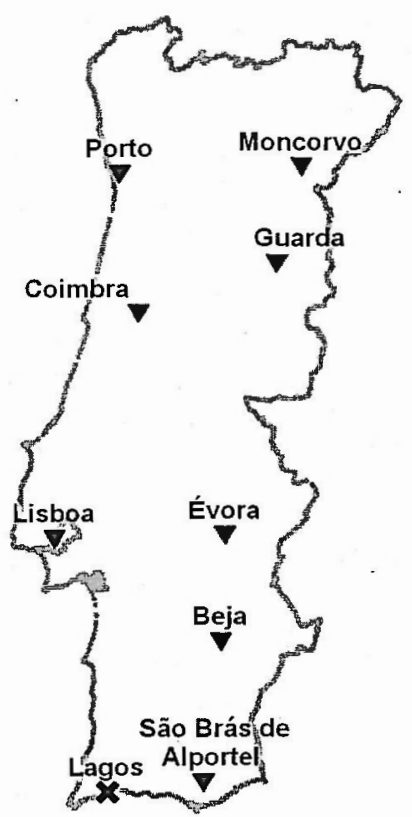

Figura 6 - Tendência da precipítação no mês de Março, em Portugal Continental, no período 1900-2000, para um nível de confiança mínimo de $95 \%$ : tendência crescente $(\boldsymbol{A})$, tendência decrescente $(\boldsymbol{\nabla})$ e sem tendência $(\boldsymbol{X})$. também observada, para dados de Portugal Continental, por e.g. PORTELA eQUINTELA (1998), SERRANO et al. (1999), MIRANDA et al. (2002) e de LIMA et al. (2005), embora para períodos de observação mais curtos no século XX. Fenómeno semelhante foi verificado noutros pontos da Europa, por outros investigadores, relativamente à presença de tendência na precipitação no mês de Março. SERRANO et al. (1999) encontraram a mesma tendência decrescente no mês de Março também em estações meteorológicas espanholas localizadas sobre a região de influência Atlântica da Península Ibérica, enquanto que estações de influência Mediterrânea ou Cantábrica não apresentaram tendência significativa. Na Irlanda foi registado um aumento bastante significativo no mês de Março e também no mês de Outubro, embora não tão significativo (ver KIELY, 1999).

Para estimar o valor da tendência da precipitação mensal (mm/década) no período 1900-2000, indicada no Quadro III, fez-se também uso do método não paramétrico de Sen, considerando-se os níveis de confiança de 90, 95, 99 e 99.9\%. O Quadro 3 evidencia a tendência decrescente, muito significativa, da precipitação no mês de Março. Este decréscimo, para a precipitação no Porto, é mostrado na Figura 7, a título exemplificativo; por década, o decréscimo médio observado corresponde a $4.7 \%$ da precipitação média no mês de Março.

Observa-se também generalizadamente uma tendência decrescente na precipitação no mês de Novembro, embora só estatisticamente significativa (90\%) em Évora, onde o decréscimo médio por década corresponde a cerca de $4 \%$ da precipitação média em Novembro. O decréscimo generalizado da precipitação no mês de Março (também em certa medida observado em Fevereiro, embora sem significância estatística) e no mês de Novembro é compensado por aumentos, geralmente estatisticamente não significativos, observados principalmente na última parte do Inverno (Dezembro e Janeiro) e no

\begin{tabular}{|l|c|c|c|c|c|c|c|c|c|c|c|c|c|}
\hline Estação & Janeiro & Fevereiro & Março & Abril & Maio & Junho & Julho & Agosto & Setembro & Outubro & Novembro & Dezembro & Anual \\
\hline Porto & 3.81 & 1.1 & $-5.82^{*}$ & 1.87 & 2.92 & -0.61 & -0.48 & 0.46 & 1.53 & 2.32 & 1.36 & 0.05 & 11.51 \\
\hline Moncorvo & 1.09 & -0.31 & $-2.59^{*}$ & 0.23 & -0.21 & -0.26 & 0 & 0 & -1.23 & -1.03 & -1.38 & -1.12 & -4.70 \\
\hline Guarda & -0.78 & -0.49 & $-7.54^{* *}$ & -0.55 & 1.78 & -1.50 & 0.00 & 0.21 & -0.71 & 0.35 & -3.44 & 0.47 & -16.38 \\
\hline Coimbra & 3.92 & 2.35 & $-5.39^{*}$ & 0.11 & 1.47 & -0.10 & -0.32 & -0.11 & -0.99 & 1.10 & -1.73 & -1.74 & -4.41 \\
\hline Lisboa & 1.97 & 0.28 & $-3.31^{*}$ & 0.41 & 1.04 & -0.34 & 0.00 & 0.00 & -0.83 & -0.61 & -1.78 & -0.26 & 0.84 \\
\hline Évora & 1.03 & -1.42 & $-4.10^{* *}$ & 1.04 & 0.55 & -0.69 & 0.00 & 0.00 & 0.27 & -0.08 & $-3.43^{+}$ & -0.12 & -4.34 \\
\hline Beja & 1.37 & -0.48 & $-3.28^{*}$ & 1.00 & -0.23 & -0.22 & 0.00 & 0.00 & 0.00 & 0.15 & -1.73 & 1.76 & -0.85 \\
\hline Lagos & 1.27 & 0.34 & -1.58 & 1.23 & 0.29 & $-0.10^{+}$ & 0.00 & 0.00 & 0.00 & 0.38 & -1.85 & 1.38 & 8.49 \\
\hline S. B.Alportel & 4.27 & -1.78 & $-5.37^{*}$ & 0.99 & 1.23 & $0.03^{*}$ & 0.00 & 0.00 & $0.40^{* *}$ & 0.00 & -0.28 & $6.21^{+}$ & 8.00 \\
\hline
\end{tabular}




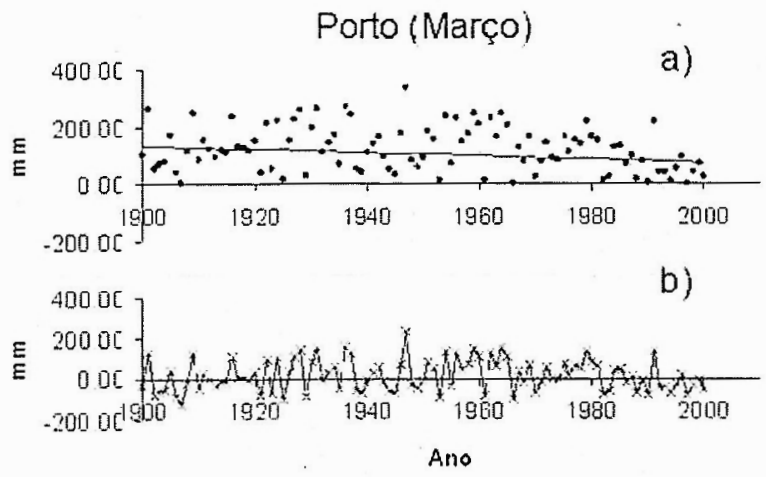

Figura 7 - Precipitação (em mm) no mês de Março, no Porto, no período 1900-2000: a) Série parcial e respectiva recta de tendência com declive obtido com o estimador de Sen;

b) resíduos da precipitação no mês de Março em cada ano, relativamente à precipitação média nesse mês.

final da Primavera (Abril e Maio). A tendência crescente da precipitação no mês de Dezembro só é estatisticamente significativa em São Brás de Alportel, para um nível de confiança de $90 \%$, e corresponde, por década, a cerca de $4 \%$ da precipitação média nesse mês. Observa-se também umatendência crescente da precipitação em Outubro, em 6 das 9 localizações estudadas.

Alguns estudos têm sido dedicados ao estabelecimento de relações quantitativas entre a precipitação mensal e o índice NAO - North Atlantic Oscillation (e.g. HURREL, 1995; ULBRICH etal., 1999; TRIGO e PALUTIKOF, 2001; RODRÍGUEZ-PUEBLA et al., 2001; KNIPPERTZ et al., 2003). Alterações ao nível da circulação atmosférica têm afectado a precipitação observada em diferentes regiões daEuropa (e.g BARDOSSY e CASPARY, 1990; TRIGO et al, 2002). Estas alterações têm conduzindo em determinadas alturas do ano e nalguns locais a decréscimos naprecipitação (como tem sidoobservado, por exemplo, em Março no Sul da Europa), enquanto noutros locais se observam aumentos (como, por exemplo, na mesma altura do ano no Norte da Europa; e.g. KIELY, 1999) como foi referido acima.

\section{Considerações finais}

Neste artigo investigou-se a presença de tendência na precipitaçẫo anual e mensal em Portugal Continental, no século XX, a partir de séries temporais de 9 localizações espacialmente dispersas em Portugal Continental. Em relação à precipitação anual não foi observada tendência com significância estatística (pelo menos para um nível de confiança de 90\%). Relativamente à distribuição sazonal da precipitação os resultados mais significativos correspondem à tendência decrescente da precipitação no mês de Março observada de uma forma generalizada em todas as regiões do País, sendo também de salientar, embora não tendo a mesma expressão, o decréscimo da precipitação no mês de Novembro. A tendência decrescente da precipitação nestes meses parece ser de alguma forma compensada como a tendência crescente observada nalguns outros meses do ano (nomeadamente Dezembro/Janeiro e Abril/Maio).

As alterações observadas na distribuição das quantidades de precipitação ao longo do ano poderão afectar de forma importante a gestão dos recursos. Têm potenciais implicações no ciclo hidrológico e, portanto, em todas as actividades com ele relacionadas. As alterações na distribuição da precipitação ao longo ao ano poderão ter várias origens, que é importante explorar, para possibilitar uma avaliação das potenciais consequências, por exemplo, ao nível da ocorrência de cheias e secas. As principais dificuldades associadas a este tipo de estudos residem, principalmente, na grande variabilidade espacial e temporal de alguns dos processos relevantes, a escassez de dados com resolução adequada e a limitada duração das séries de observações.

\section{Agradecimentos}

Este trabalho foi financiado pela FCT - Fundação para a Ciência e Tecnologia, através do projecto POCTI/MGS/39039/2001 e POCTI/ECM/48619/2002. Este financiamento foi atribuído no âmbito do Eixo 2, Medida 2.3 do POCTI - Programa Operacional "Ciência, Tecnologia, Inovação" do QCA III (comparticipação FEDER). No âmbito do projecto POCTI/ /MGS/39039/2001, agradece-se a colaboração da Dra.M.FátimaE.S. Coelho, doInstitutode Meteorologia.

\section{Bibliografia}

BARDOSSY, A. e CASPARY, H. J. (1990) - "Detection of climate change in Europe by analysing European atmospheric circulation patterns from 1988 to 1989". Theor. Appl. Climat.. 42, pp. $155-167$.

de LIMA, M. I. P.(1998)-Multifractals and the temporal structure of rainfall. Ph.D. Thesis, Wageningen Agricultural Univiversity, Wageningen, The Netherlands.

de LIMA, M. I. P., SCHERTZER, D., LOVEJOY, S. e de LIMA, J. L. M. P. (2002) - "Multifractals and the study of extreme precipitation events: a case study from semi-arid and humid regions in Portugal." In SINGH, V.P., AL-RASHID, M. e SHERIFM.M. (ed.)-Surface Water Hydrology, A. A.Balkema Publishers, Swets \& Zeitlinger B.V., Lisse, The Netherlands, pp. 195-211.

de LIMA, M. I. P., MARQUES, A. C. P., de LIMA, J. L. M. P. e COELHO, M. F. E. S. (2005) - "Precipitation trends in Mainland Portugal in the period 1941-2000". Actas do Fourth 
Inter-Celtic Colloquium on Hydrology and Management of Water Resources, Guimarães (Portugal), 11-13 Julho 2005 (submetido).

GILBERT, R. O. (1987) - Statistical methods for environmental pollutionmonitoring. New York(EUA), VanNostrandReinhold.

HURREL, J. W. (1995) - "Decadal trends in the North Atlantic Oscillations: regional temperatures and precipitation". Science, 269, pp. 676-679.

KENDALL, M. G. (1948)-Rank correlation methods. Hafner, New York.

KIELY, G. (1999) - "Climate change in Ireland from precipitation and streamflow observations". Adv. in Water Resour., 23, pp. 141-151.

KNIPPERTZ, P., CHRISTOPH, M. e SPETH, P. (2003) - “Longterm precipitation variability in Morocco and the link to the large-scale circulation in recent and future climates". Meteorol. Atmos. Phys., 83, pp. 67-88.

MANN, H. B.(1945)-“Non-parametric test against trend". Econometrika, 13, pp. 245-259.

MIRANDA, P., COELHO, F. E. S., TOMÉ, A. R., VALENTE, M. A., CARVALHO, A., PIRES, C., PIRES, H.O., PIRES, V. C. e RAMALHO, C. (2002) - " $20^{\text {th }}$ Century Portuguese Climate and Climate Scenarios". In SANTOS, F. D., FORBES K. $\mathrm{e}$ MOITA, R. (ed.) - Climate Changes in Portugal: Scenarios, Impacis and Adaptation Measures, Gradiva, Lisboa, Portugal, pp. 27-83.

PORTELA, M. M. e QUINTELA, A. C. (1998) - "Indícios de mudança climática em séries de precipitação em Portugal Continental". Recursos Hidricos, APRH, 19(2,3), pp. 41-74.
SEN, P. K. (1968) - "Estimates of the regression coefficient based on Kendall's tau". J. of the American Statistical Association, 63, pp. 1379-1389.

SERRANO, A., MATEOS, V. L. e GARCIA, J. A. (1999) - "Trend analysis of monthly precipitation over the Iberian Peninsula for the period 1921-1995". Phys. Chem. Earth, 24, pp. 85-90.

SNEYERS, R. (1990) - On the statistical analysis of series of observations, WMO Technical Note $\mathrm{N}^{\circ} 143$, World Meteorological Organization.

RODRÍGUEZ-PUEBLA, C., ENCINAS, A. H. e SÁENZ, J. (2001) - "Winter precipitation over the Iberian peninsula and its relationship to circulation indices". Hydrology and Earth System Sciences, 5(2), pp. 233-244.

TRIGO, R.M. e PALUTIKOF, J.P.(2001) - "Precipitation scenarios over Iberia: a comparison between direct GCM output and different downscaling techniques". J. of Climate, 14, pp. 4422-4446.

TRIGO, R. M., OSBORN, T. J. e CORTE-REAL, J. M. (2002) "The North Atlantic Oscillation influence on Europe: climate impacts and associated physical mechanisms". Climate Res., 20, pp. 9-17.

ULBRICH, U., CHRISTOPH, M., PINTO, J. G. e CORTE-REAL, J. (1999) - "Dependence of winter precipitation over Portugal on NAO and baroclinic wave activity". Intern. $J$. of Climatology, 19(4), pp. 379-390.

WALD, A.e WOLFOWITZ,J.(1943)_"An exact test for randomness in the non-parametric case based on serial correlation". Annals Mathematic and Statistics, 14, pp. 378-388. 